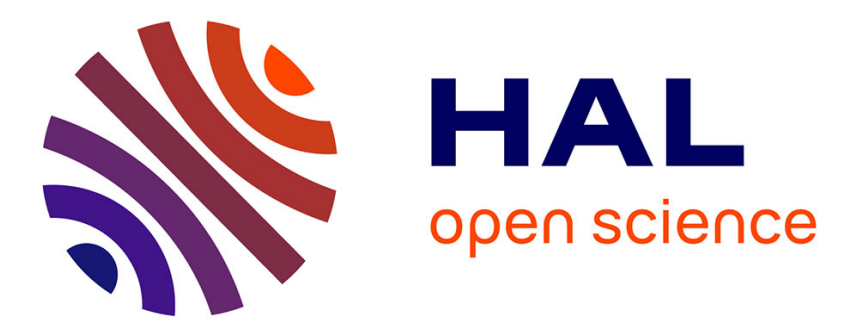

\title{
Prior intention can locally tune inhibitory processes in the primary motor cortex: direct evidence from combined TMS-EEG
}

\author{
M Bonnard, L Spieser, H B Meziane, J B de Graaf, J Pailhous
}

\section{- To cite this version:}

M Bonnard, L Spieser, H B Meziane, J B de Graaf, J Pailhous. Prior intention can locally tune inhibitory processes in the primary motor cortex: direct evidence from combined TMS-EEG. European Journal of Neuroscience, 2009, 30, pp.913 - 923. 10.1111/j.1460-9568.2009.06864.x . hal-01453473

\author{
HAL Id: hal-01453473 \\ https://hal.science/hal-01453473
}

Submitted on 22 Nov 2017

HAL is a multi-disciplinary open access archive for the deposit and dissemination of scientific research documents, whether they are published or not. The documents may come from teaching and research institutions in France or abroad, or from public or private research centers.
L'archive ouverte pluridisciplinaire $\mathbf{H A L}$, est destinée au dépôt et à la diffusion de documents scientifiques de niveau recherche, publiés ou non, émanant des établissements d'enseignement et de recherche français ou étrangers, des laboratoires publics ou privés. 


\title{
Prior intention can locally tune inhibitory processes in the primary motor cortex: direct evidence from combined TMS-EEG
}

M. Bonnard, L. Spieser, H. B. Meziane, J. B. de Graaf and J. Pailhous

Mediterranean Institute of Cognitive Neuroscience 'INCM', UMR 6193, CNRS-University of Aix-Marseille, 31 Chemin Joseph Aiguier, 13402 Marseille, Cedex 20, France

Keywords: combined TMS-EEG, intention, motor cortex

\begin{abstract}
Human subjects are able to prepare cognitively to resist an involuntary movement evoked by a suprathreshold transcranial magnetic stimulation (TMS) applied over the primary motor cortex (M1) by anticipatory selective modulation of corticospinal excitability. Uncovering how the sensorimotor cortical network is involved in this process could reveal directly how a prior intention can tune the intrinsic dynamics of M1 before any peripheral intervention. Here, we used combined TMS-EEG to study the cortical integrative processes that are engaged both in the preparation to react to TMS (Resist vs. Assist) and in the subsequent response to it. During the preparatory period, the contingent negative variation (CNV) amplitude was found to be smaller over central electrodes (FC1, C1, $\mathrm{Cz}$ ) when preparing to resist compared with preparing to assist the evoked movement whereas $\alpha$-oscillation power was similar in the two conditions. Following TMS, the amplitude of the TMS evoked-N100 component was higher in the Resist than in the Assist condition for some central electrodes ( $\mathrm{FCz}, \mathrm{C} 1, \mathrm{Cz}, \mathrm{CP} 1, \mathrm{CP} 3)$. Moreover, for six out of eight subjects, a single-trial-based analysis revealed a negative correlation between CNV amplitude and N100 amplitude. In conclusion, prior intention can tune the excitability of M1. When subjects prepare to resist a TMS-evoked movement, the anticipatory processes cause a decreased cortical excitability by locally increasing the inhibitory processes.
\end{abstract}

\section{Introduction}

Studying preparatory motor sets offers a privileged way to investigate the interaction between cognitive and sensorimotor functions (Evarts et al., 1984; Requin et al., 1991; Georgopoulos, 2000). Indeed, this gives the opportunity to study how cognitive processes might interact with motor processes prior to the interaction between central and peripheral mechanisms occurring during motor execution (Day et al., 1991; Palmer \& Ashby, 1992). For several years, both in humans and in monkeys, strong neurophysiological evidence brought to the fore the idea that cognitive and motor functions are so intimately linked during motor preparation that cognitive factors can set neural activity in motor-related cortical areas, even in the primary motor cortex (M1) (for reviews, see Evarts et al., 1984; Requin et al., 1991; Georgopoulos, 2000; Riehle, 2004; Bonnard et al., 2004). As in all complex systems, perturbing the dynamic state of the brain allows us to understand its dynamics from the way it reacts to this perturbation (Jackson et al., 2002; Meyer-Lindenberg et al., 2002; Massimini et al., 2005). Moreover, in the case of living systems which have intentionality, relating this reaction to the way the brain has prepared to react to the perturbation as a function of

Correspondence: Dr M. Bonnard, as above.

E-mail: mireille.bonnard@incm.cnrs-mrs.fr

Received 18 May 2009, revised 23 June 2009, accepted 25 June 2009 prior intention has been found particularly fruitful in the study of cognitive-motor interactions (Evarts \& Tanji, 1974; Tanji \& Evarts, 1976). Indeed, this procedure reveals to what extent a prior intention can anticipatorily tune the intrinsic dynamics of the brain. For example, using microelectrode recordings in the pre-central motor cortex, Evarts \& Tanji (1974) demonstrated anticipatory activity of numerous motor cortex neurons after a prior instruction indicating the animal how to respond (push or pull) to a subsequent mechanical perturbation that triggered the instructed movement. For most of the recorded neurons, this activity modulation differed according to the instruction (Tanji \& Evarts, 1976). In addition, the short latency motor cortex response $(20 \mathrm{~ms})$ evoked by the subsequent perturbing stimuli differed markedly depending upon the prior instruction (Evarts \& Tanji, 1974).

Transcranial magnetic stimulation (TMS) is a powerful tool that can directly temporarily perturb brain dynamics in healthy human subjects (for studies in the field of sensorimotor control see Ilmoniemi et al., 1997; Paus et al., 2001; Meyer-Lindenberg et al., 2002; Kimura et al., 2006). Regarding cognitive-motor interactions, several recent TMS studies (Bonnard et al., 2003; Sohn et al., 2003; Camus et al., 2004) were designed to dissociate the influence of cognitive (intention-related) processes from that of motor (performance-related) processes in the tuning of corticospinal (CS) excitability. A suprathreshold TMS pulse was applied over the left primary motor cortex in order to evoke a right- 
handed movement while the subjects were instructed to prepare mentally (i.e. without changing their ongoing EMG activity) either to 'let go' or to 'resist' this TMS-evoked movement (Bonnard et al., 2003). Compared with peripheral perturbations (Evarts \& Tanji, 1974; Rothwell et al., 1982), such central perturbations offer a unique opportunity to activate the primary motor cortex and its descending motor tracts (CS tract among others) and to obtain a measurement of cortical (and/or CS) excitability before the movement is actually produced, i.e. before any influence of afferent inflow induced by the evoked movement. Therefore, one can isolate the influence of top-down processes over the dynamic state of the primary motor cortex (and/or of the CS tract). These studies revealed a remarkable capability of human beings: to their great surprise, subjects were able to cognitively prepare themselves to resist a TMS-evoked movement without changing their ongoing EMG activity. Our previous work has shown that this was achieved by a selective anticipatory modulation of CS excitability (Bonnard et al., 2003), in the sense that motor-evoked potentials (MEPs) in the prime mover muscle were smaller when subjects resisted the TMSevoked movement. Moreover, in the resist condition, this instruction set also lengthened the duration of the cortical silent period (SP), which presumably relates to intra-cortical inhibition (Mathis et al., 1998). Interestingly, similar results on CS excitability were obtained by Sohn et al. (2003) who asked their subjects either to imagine squeezing hands (positive imagery) or to imagine suppression of TMS-evoked twitching movement (negative imagery). Compared with a rest condition without imagination, the authors reported a decreased MEP amplitude in the prime mover muscle in negative motor imagery, indicating a lowered excitatory CS drive.

In order to test the cortical origins of these modulations, Sohn et al. (2003) applied paired pulse TMS over the primary motor cortex. No significant change either in intracortical inhibition or in intracortical facilitation during such imagination of suppressing movement was found, however. In other words, no suggestions regarding the cortical mechanisms underlying this cognitive tuning of CS excitability were found. In healthy human subjects, combined TMS-EEG today offers a unique opportunity to study not only how the brain responds to TMS (Ilmoniemi et al., 1997; Paus et al., 2001; Nikulin et al., 2003), but also to relate this response to the way the brain has prepared to a TMS pulse. The present experiment was designed from this perspective in order to investigate the cortical integrative processes engaged in the preparation to resist a TMS-evoked movement and in the subsequent response to TMS. We aimed to establish how subtle changes in cortical excitability might be related to anticipatory processing in the sensorimotor cortex. Cortical anticipatory processes preceding TMS were investigated using both contingent negative variation (CNV) for the evaluation of cortical excitability (Bastiaansen et al., 1999; Brunia \& van Boxtel, 2001) and $\alpha$-oscillation power for the evaluation of cortical activation (Pfurtscheller \& Lopes da Silva, 1999; Filipović et al., 2001; Babiloni et al., 2003). Indeed, CNV is known to reflect an anticipatory increase in cortical excitability (Bastiaansen et al., 1999) whereas an event-related decrease in $\alpha$-band oscillatory activity, called event-related desynchronization $(\alpha$-ERD), is associated with an activated state of the underlying areas (Lopes da Silva \& Pfurtscheller, 1999; Filipović et al., 2001; Babiloni et al., 2003). The brain response to TMS was investigated by measuring the TMS-evoked potential. We focused on the N100 component, which reflects inhibitory processes (Nikulin et al., 2003; Bender et al., 2005). We investigated whether (i) during cognitive preparation to TMS, CNV late wave and $\alpha$-oscillation power differed according to the prepared motor response to TMS; (ii) following TMS, the TMS-evoked N100 component differed according to the motor response to TMS; and (iii) modulations in cortical excitability anticipating the TMS pulse (as revealed by $\mathrm{CNV}$ ) are related to inhibitory processes (as revealed by the the TMS-evoked N100 component).

\section{Material and methods \\ Subjects}

This study was conducted on eight healthy right-handed subjects (four males, four females), from 22 to 42 years old, with their full informed consent. None had neurological antecedents, or any contraindication for TMS, and all were familiarized with the TMS and the experimental protocol. This study was approved by the local ethical committee (CPP Sud-Méditterranée II) and was in accordance with the declaration of Helsinki.

\section{Experimental set-up}

The subject sat in a comfortable reclining armchair, facing a computer screen, on which the instructions appeared. A pillow under-vacuum placed around the neck reduced head movement. The subject's right forearm was in a supine position and attached to an armrest (see Fig. 1). The armrest was adapted for each subject so that the elbow was at an angle of approximately $110^{\circ}$ and the hand lying out of the armrest. The forearm was fixed in order to limit movements in the horizontal plane and to allow only flexion and extension of the wrist. An easily graspable object was placed in the right hand, in order for it to maintain a half-closed position. In the resting position, the hand laid on a support slightly lower than the armrest such that the wrist was positioned in an extended position of approximately $30^{\circ}$.

\section{TMS}

We used a Magstim 200 stimulator (Magstim Co., Whitland, UK) generating a monophasic magnetic field of up to $1.7 \mathrm{~T}$, connected to a coplanar figure-of-eight coil with external loop diameter of $9 \mathrm{~cm}$. The coil was maintained in the desired position by a custom-made fixation system consisting of a knee-joint, connected to a sliding system in the horizontal plane (cf. Fig. 1). The stimulation system was connected to a neuro-navigation device (Navigation Brain System, Nexstim, Helsinki, Finland) which used the anatomical magnetic resonance image (MRI) of each subject to guide stimulation in a precise way. The system calculates an estimate of the electric field induced in the cortex by the TMS in real time on the anatomical MRI of the subject (cf. Fig. 3B). Using this neuro-navigation system, the coil was placed such that it stimulated the left central sulcus at the location of the omega that corresponds to the cortical representation of the right hand in M1 (Rumeau et al., 1994; Yousry et al., 1997; Sastre-Janer et al., 1998). The handle was pointing backward and laterally approximately $45^{\circ}$ with respect to the midline in order to have the current direction perpendicular to the central sulcus. In case of head movement during the experiment, we interrupted the session to reposition the coil precisely. The stimulation intensity was adjusted for each subject such that TMS evoked a clear movement of wrist flexion; the mean stimulation intensity for the group $( \pm \mathrm{SD})$ was $72 \pm 11 \%$ of the maximum stimulation intensity corresponding to $120 \%$ of the rest motor threshold. [The motor threshold corresponds to the stimulation intensity resulting in an MEP of more than $50 \mu \mathrm{V}$ in the flexor carpi radialis (FCR) muscle in about half of the trials.] The subjects wore earplugs and head-phones which delivered white noise to reduce the chances of hearing the coil discharge-induced click. 

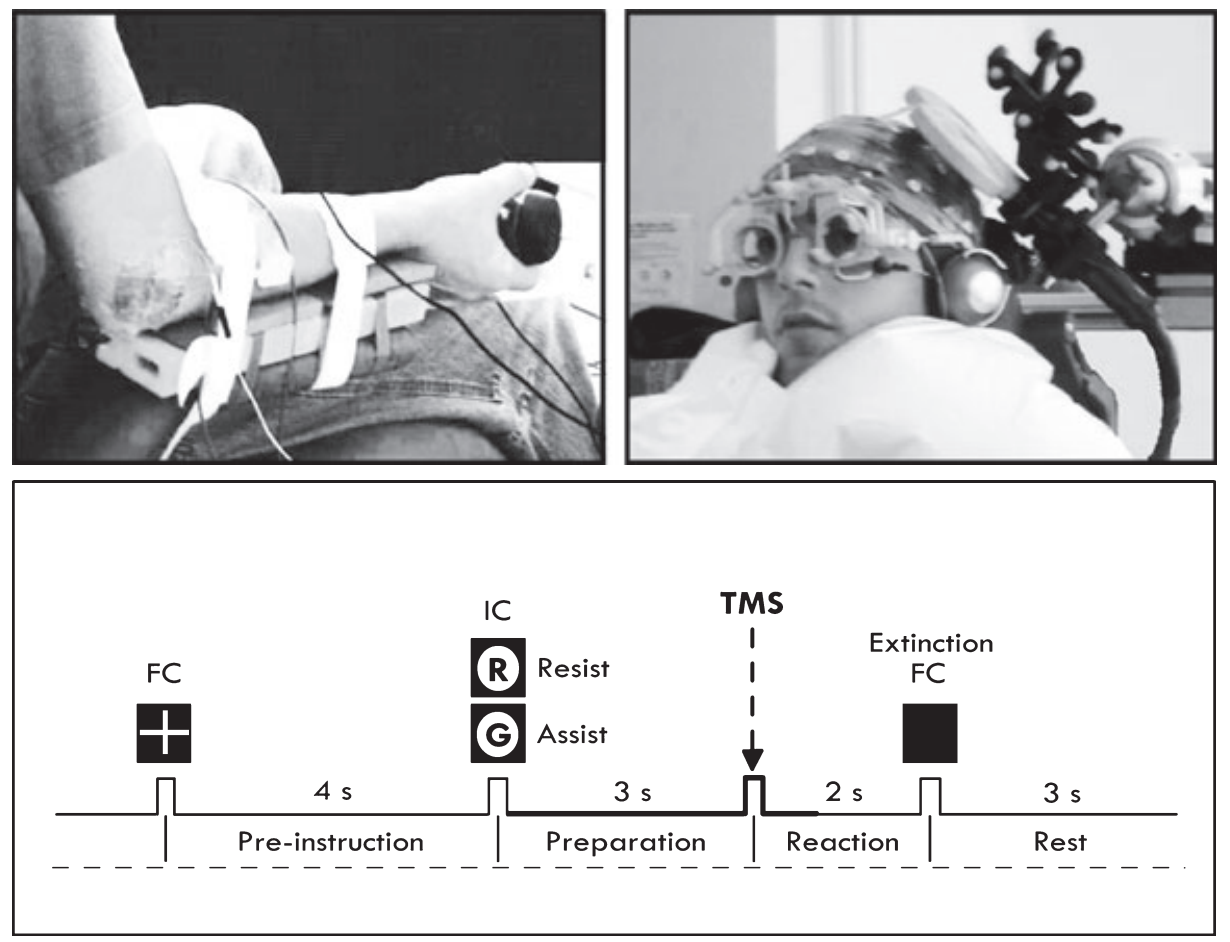

FIG. 1. Experimental set-up and protocol. Left: the subject's right forearm was attached to an armrest, in a supine position. An easily grabbable object was placed in the hand, in order for it to maintain a half-closed position. In the resting position, the hand laid on a support slightly lower than the armrest. Right: using a neuronavigation system which used the anatomical MRI of each subject to guide stimulation, the coil was placed so it stimulated the hand area in the left primary motor cortex. The coil was maintained by a fixation system. The glasses, equipped with markers, allowed the neuro-navigation system to detect head movements. Below: time course of the events occurring during a trial (see text). FC, fixation cross; IC, instruction cue; R, red circle; G, green circle.

\section{Recording data}

The EEG was recorded continuously with TMS-compatible EEG equipment ('high-density' amplifiers, Advanced Neuro Technology, Enschede, Netherlands). The equipment contains full-band EEG DC amplifiers (no filtering was applied during acquisition). After the TMS pulse, the amplifiers return to physiological signal level within milliseconds (see Fig. 2). We used a 64-electrode cap ('WaveGuard', ANT) with sintered $\mathrm{Ag} / \mathrm{AgCl}$ electrode material, mounted on an elastic cap positioned according to the 10-20 method extended to 64 electrodes. The material and shape of the electrodes as applied in the 'waveguard' cap are designed for optimal stability of incoming signal. This cap is designed with an active shielding of each lead (i.e. the electrode inputs are shielded with the electrode signal itself) which limits electrical noise. The ground electrode was positioned on the forehead. The ANT-system uses an averaged reference for all the electrodes. Skin-electrode impedance was maintained below $5 \mathrm{k} \Omega$.

The electrooculogram related to eye blinks were recorded using bipolar electrodes. The EMG signals from the FCR and extensor carpi radialis (ECR) muscles (flexor and extensor of the wrist, respectively) of the right forearm were recorded using bipolar surface electrodes and subsequently band-pass filtered $(3-450 \mathrm{~Hz})$. An electrogoniometer (Biometrics) measured the angular displacement of the right wrist. The data and event triggers were simultaneously and continuously sampled at $1024 \mathrm{~Hz}$.

Parameters such as the localization, orientation and intensity of the induced electrical field, associated with each TMS pulse, were recorded by the neuro-navigation device (cf. Fig. 3B). For all subjects, the maximal induced electrical field was on average $( \pm \mathrm{SD})$ $90.4 \pm 28.2 \mathrm{~V} / \mathrm{m}$. It is important to note that for each subject the induced electrical field was stable throughout the experiment; the standard deviation corresponds to inter-subject differences.

\section{Protocol}

Subjects had to prepare cognitively for a wrist movement evoked by a suprathreshold TMS applied over M1 (as in Bonnard et al., 2003). They were instructed to prepare mentally (i.e. without changing their ongoing EMG activity) either to 'Resist' the TMS-evoked movement or to 'Assist' the TMS-evoked movement. The experiment consisted of four recording sessions, including 50 trials each. Figure 1 presents the time course of the different events (controlled by a Labview interface) occurring during a trial. At the beginning of each trial, the hand of the subject was at rest, with the back of the hand leaning on the support. Trials began with the appearance of a fixation cross in the middle of the screen. Upon its appearance, the subject had to align his/her hand with the forearm; this required a small contraction of the wrist flexor to compensate for the gravitational force. (An experimenter verified that this initial position remained the same throughout the experiment.) The instruction 'resist' (RES) or 'assist' (ASS) the TMS-evoked movement appeared $4 \mathrm{~s}$ later as a red or a green circle, respectively, for $500 \mathrm{~ms}$. After the instruction cue (IC) was switched off, the fixation cross reappeared. The subject was instructed to carry out the task without modifying muscle activity at the wrist, for example by co-contracting the antagonist muscle groups when preparing himself/herself to resist. He/she had to prepare mentally to resist or assist the evoked movement. The absence of co-contraction was visually checked on-line by an experimenter and further verified by off-line analysis of the EMG activity recorded during the preparatory period. Three seconds after the appearance of the IC, TMS was triggered. Two seconds following TMS, the fixation cross 

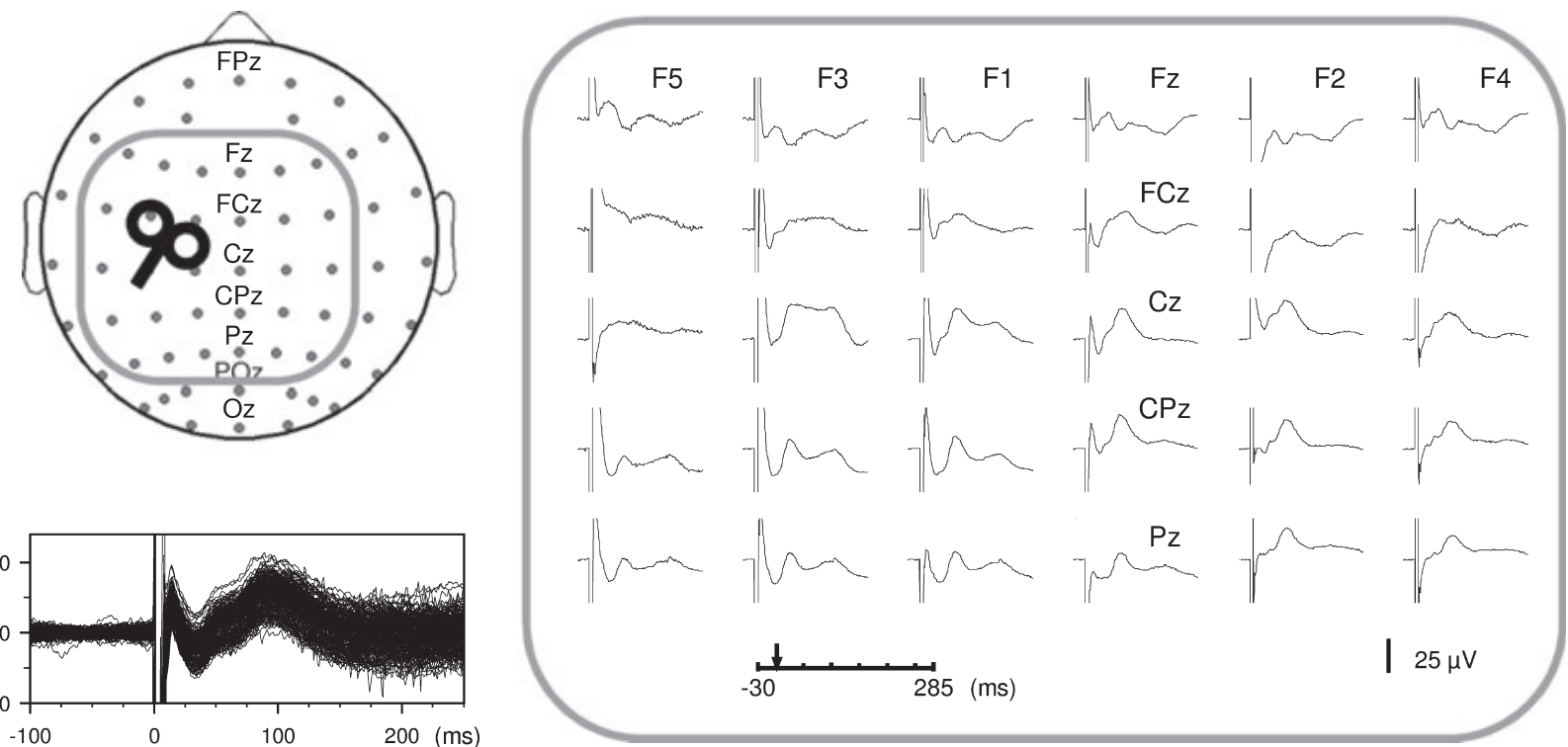

FIG. 2. Right: averaged EEG response to TMS (52 trials) at scalp locations close to the stimulation site (outlined in grey on top left). Negativity is upward. Bottom left: 196 single traces of the same subject recorded over $\mathrm{Cz}$, in response to TMS occurring at time 0.
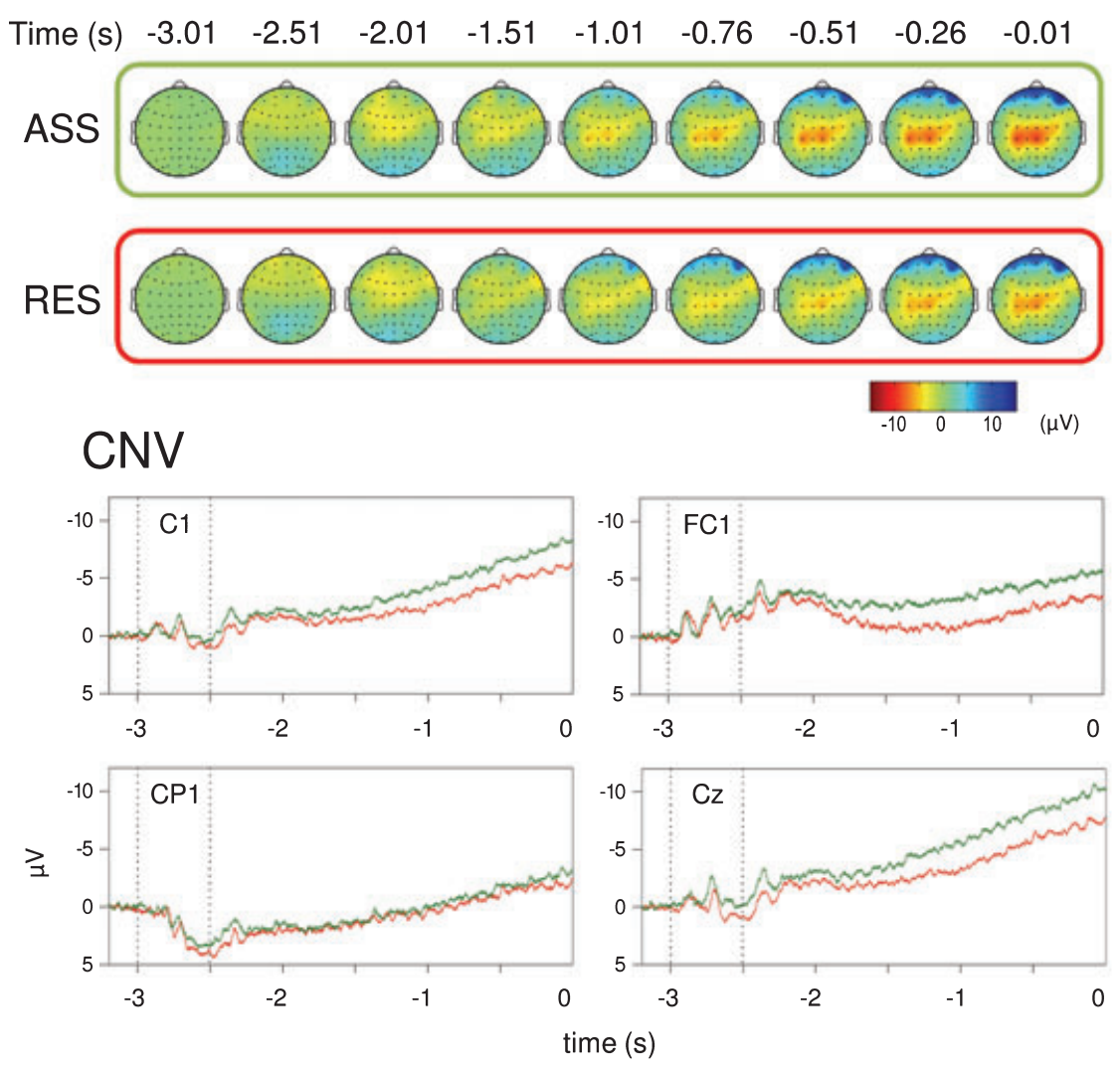

\section{ERP}
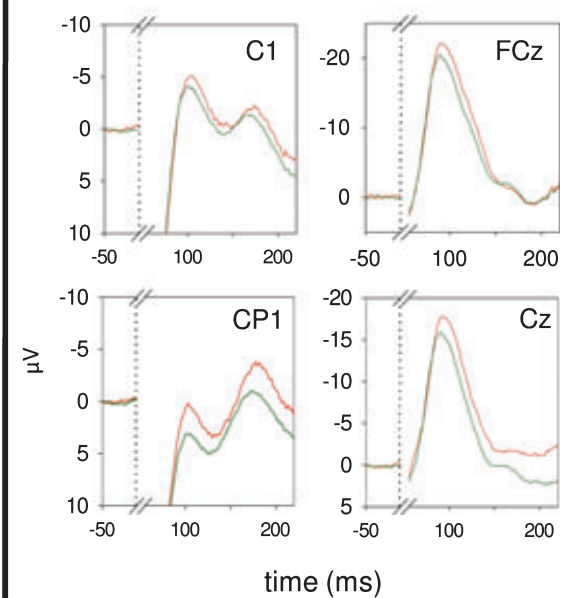

FIG. 3. Grand average EEG activity observed in each condition preceding TMS (A) and following TMS (B). (A) Top: topographical maps during the preparatory period for the ASS and RES conditions. TMS occurred at $0 \mathrm{~s}$. Bottom: CNV plots for four central electrodes during the preparatory period. Notice that no condition effect was observed for CP1. Green, ASS; red, RES. The vertical dotted lines represent the instruction onset and offset. (B) Top: colour map of the electrical field induced by TMS in one trial. Bottom: ERP in response to TMS. Grand average potentials are shown for four central electrodes. The time axis is interrupted from 0 to $55 \mathrm{~ms}$. 
was removed, and the subject relaxed and returned to the rest position for $3 \mathrm{~s}$, before starting a new trial. RES and ASS trials were presented randomly during the four sessions (25 ASS and 25 RES per session, on average), and the order of the four sessions was randomized. Between two sessions, subjects rested for a few minutes.

\section{Data analysis}

\section{Behavioural and EMG data}

We first verified that the behavioural and muscle responses to TMS (MEPs and SP) were congruent with those reported in the literature (Mathis et al., 1998; Bonnard et al., 2003; Sohn et al., 2003). Concerning the behavioural data, the amplitude of the evoked movement observed over all trials was found to differ between ASS and RES conditions $(P<0.05)$. For the analysis of the muscle response to TMS, we used the same procedure reported by Bonnard et al. (2003). In brief, the data were sorted on the evoked-movement amplitude. Trials that did not show a clear wrist flexion in the ASS condition, or inversely showed a large deviation from the initial position in the RES condition, were removed. On average, the proportion of remaining trials was $65 \pm 16 \%$ for the ASS condition and $71 \pm 17 \%$ for the RES condition. Then, for each subject, we eliminated all trials whose initial EMG level during the preparation did not fall within the common range for the two conditions (Schieppati et al., 1996). For the selected trials $(51 \pm 13$ trials per subject for the ASS condition and $57 \pm 13$ trials for the RES condition), the pre-TMS EMG level (mean rectified EMG value during the last $100 \mathrm{~ms}$ prior to TMS) in the flexor and extensor muscles was similar in the two conditions ( $t=0.98$, NS for the flexor; $t=-1.40$, NS for the extensor). In both muscles (FCR, ECR) and for each selected trial, the MEP amplitude was measured as the difference between the lowest and highest value of the raw EMG signal within a 20- to 50-ms time window following TMS and then averaged in each condition. For the FCR, the duration of the silent period was also measured in each trial as the time interval between the TMS and the first wave characterizing the restart of the EMG burst. For these parameters, comparisons were performed using a Student's $t$-test for paired data.

\section{EEG data}

For the EEG data, we did not apply the performance-based selection; only trials with artefacts (blinks, muscle artefacts, inadvertent motor acts, instrumental artefacts) during the preparation or the early reaction to TMS were removed. On average, we had $77 \pm 15$ trials per subject for the ASS condition and $81 \pm 15$ trials for the RES condition. We separately processed brain potentials during the preparatory period (from the $3 \mathrm{~s}$ between the onset of the IC and the occurrence of TMS) and the brain response to the TMS.

\section{Preparatory period}

\section{CNV during preparatory period}

For each subject in each condition, EEG signals were averaged across trials from $200 \mathrm{~ms}$ before the instruction onset (i.e. the coloured circle) until TMS occurrence. A baseline correction was applied to the averaged potential; the baseline was taken between 200 and $100 \mathrm{~ms}$ before the onset of the instruction ASS or RES. We then performed a grand average over the individual data in each condition using ASA (Advanced Source Analysis, ANT, Enschede, Netherlands). For each subject and each condition, the CNV amplitude was calculated as the mean value of the EEG signal obtained during the last $100 \mathrm{~ms}$ of the preparatory period (i.e. prior to the TMS pulse). For electrodes FC1, $\mathrm{FCz}, \mathrm{C} 1$ and $\mathrm{Cz}$ that showed a well-pronounced $\mathrm{CNV}$ for all subjects, $\mathrm{CNV}$ amplitudes were then compared by a two-way ANOVA for repeated measures, including the factors electrode $(\mathrm{FC} 1, \mathrm{FCz}, \mathrm{C1}, \mathrm{Cz})$ and condition (ASS, RES). All pairwise multiple comparisons were performed using a Tukey test.

\section{$\alpha$-Oscillations during preparatory period}

A time-frequency analysis was performed from $1 \mathrm{~s}$ before the instruction to $0.5 \mathrm{~s}$ before TMS. To represent the time-frequency distribution of the power during this period, we used the spectrogram based on a short-time Fourier transform of the signal calculated with a sliding window (Hamming window of 1024 samples, corresponding to $1 \mathrm{~s})$, every 64 samples $(62.5 \mathrm{~ms})$. In each subject, the spectrogram was computed for each trial, and then averaged for each condition (see Fig. 4A). Then, in order to determine $\alpha$-band frequencies for each subject, we performed a fast Fourier transform (FFT) accross the whole preparatory period. The $\alpha$-band was determined as the frequencies showing a power peak in the $\alpha$-range $(10.4 \pm 0.9 \mathrm{~Hz}$ with a bandwidth of $3.5 \pm 0.8 \mathrm{~Hz})$. In order to visualize any $\alpha$-event-related desynchronization (i.e. a decrease in power in the $\alpha$-band), the $\alpha$-band power was normalized to a baseline defined as the mean $\alpha$-band power during the last second prior to the IC (see Fig. 4B). As shown in Fig. 4C, $\alpha$-ERDs were maximal for electrodes $\mathrm{C} 5, \mathrm{C} 3, \mathrm{C} 1, \mathrm{CP} 5, \mathrm{CP} 3$ and $\mathrm{CP} 1$, and these electrodes were chosen for further analysis. In order to analyse $\alpha$-oscillation power during the last second prior to TMS and the last second prior to the IC, power spectral densities were calculated in these time windows for each trial and subsequently averaged for each condition (cf. Fig. 4D). This mean $\alpha$-power was subjected to a three-way ANOVA for repeated measures with conditions (ASS, RES), time-periods (pre-IC, pre-TMS) and electrodes $(\mathrm{C} 5, \mathrm{C} 3, \mathrm{C} 1, \mathrm{CP} 5, \mathrm{CP} 3$, and $\mathrm{CP} 1)$ as factors.

\section{Brain response to TMS}

Figure 2 shows the average EEG response to TMS for a typical subject for the central electrodes, as well as the superimposed responses observed over $\mathrm{Cz}$ in all trials of the same subject, which shows the high reproducibility of these responses.

To study the TMS-evoked response, we averaged the EEG signals across trials from $100 \mathrm{~ms}$ preceding TMS to $500 \mathrm{~ms}$ following it. A baseline correction (taken as the $100 \mathrm{~ms}$ preceding the TMS) was applied on the averaged potentials. We then performed a grand average across subjects. The central electrodes $\mathrm{FC} 1, \mathrm{FCz}, \mathrm{C} 1, \mathrm{Cz}, \mathrm{CP} 1, \mathrm{CP} 3$ and $\mathrm{CPz}$ showed a well-pronounced N100 for all subjects (see Fig. 3B). We therefore focused on the N100 amplitude on these electrodes. For each subject and each condition, the N100 amplitude was determined as the highest negative peak in the interval $80-150 \mathrm{~ms}$ after TMS. N100 amplitudes were then compared by a two-way ANOVA for repeated measures with electrode ( $\mathrm{FC} 1, \mathrm{FCz}, \mathrm{C} 1, \mathrm{Cz}, \mathrm{CP} 1, \mathrm{CP} 3, \mathrm{CPz})$ and condition (ASS, RES) as factors. All pairwise multiple comparisons were performed using a Tukey test.

In addition, electrode $\mathrm{Cz}$ showed the greatest differences between ASS and RES for both CNV and N100 amplitudes. Therefore, for this electrode, we investigated whether the CNV amplitude and N100 amplitude were correlated, regardless of condition. This analysis was conducted within subjects on the basis of single-trial analysis. In each trial, we determined CNV and N100 amplitudes in the same way as for the averaged potential analysis. 
6 M. Bonnard et al.

\section{A}

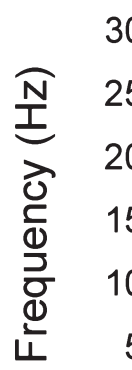
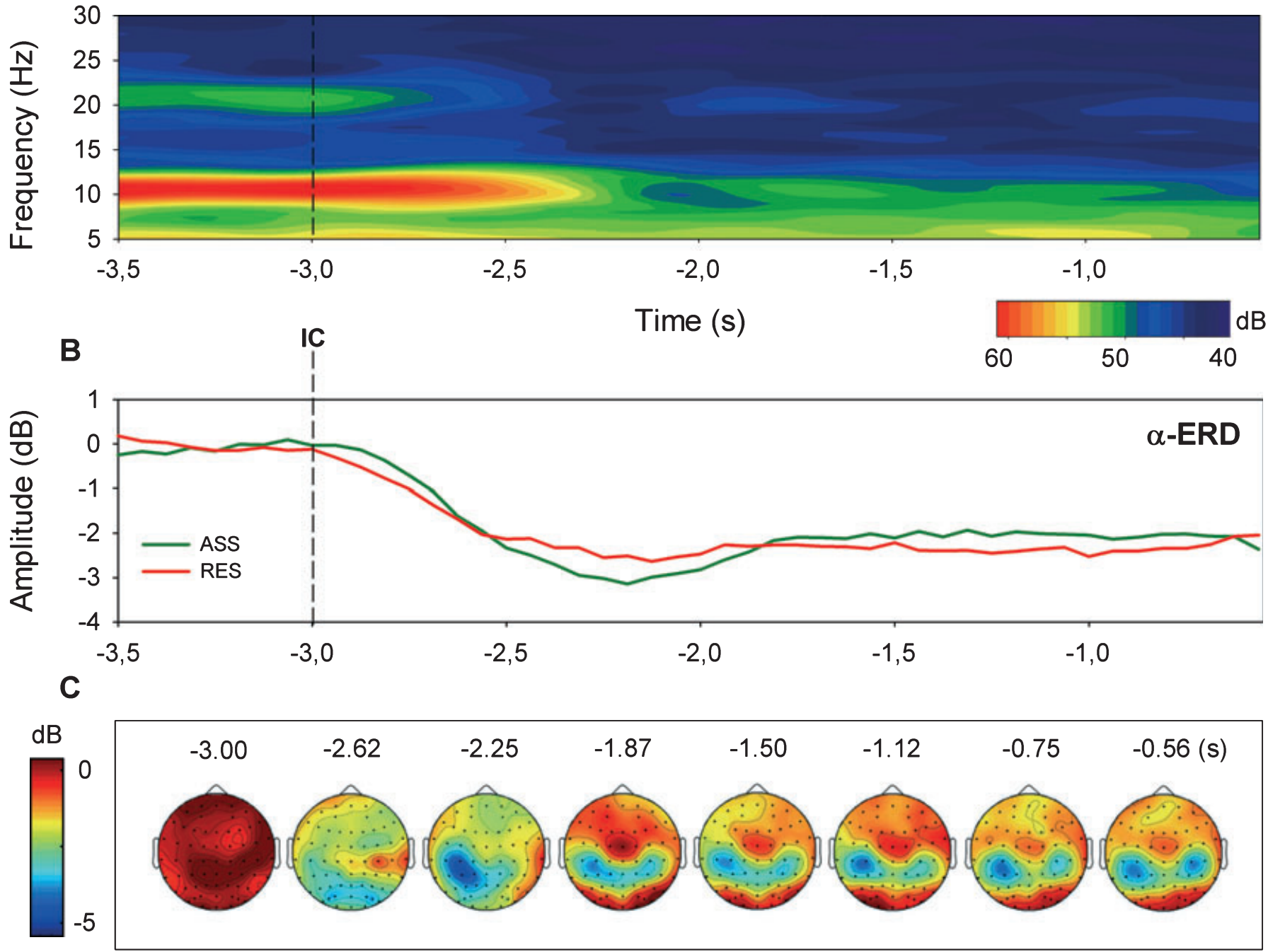

D

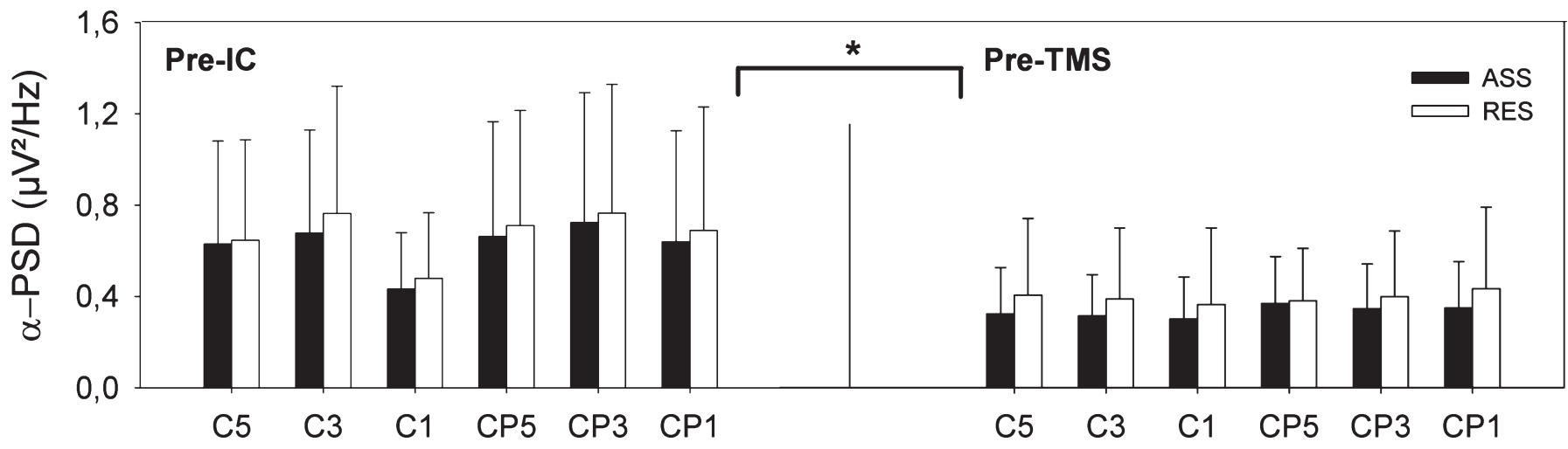

FIG. 4. (A) Trial-averaged spectrogram for one subject on electrode $\mathrm{C} 3$ in the assist condition. The IC arrived at $-3 \mathrm{~s}$, and TMS at $0 \mathrm{~s}$. (B) Grand average of $\alpha$-ERD as a function of time on electrode C3 in both ASS and RES conditions. (C) Grand average of the topographies for the evolution of $\alpha$-ERD during the preparatory period in the resist condition. Colouring of the first topographic map $(-3 \mathrm{~s})$ is due to the baseline correction (see text). Notice the clear $\alpha$-ERD on several central electrodes. (D) Mean power spectral density observed in each condition on electrodes C5, C3, C1, CP5, CP3 and CP1 in two time windows: $1 \mathrm{~s}$ pre-IC and $1 \mathrm{~s}$ preTMS. The difference between pre-IC and pre-TMS alpha oscillation power was significant (as indicated by the asterisk). 


\section{Results}

\section{Task realization}

The mean amplitude of the evoked movement was smaller in the RES than in the ASS condition (ASS: $12.1 \pm 5.8^{\circ}$; RES: $2.5 \pm 0.97^{\circ}$, $\left.t_{7}=4.58, P<0.05\right)$. For similar pre-TMS EMG levels in both conditions, in the flexor muscle, MEP amplitude was smaller in the RES compared with ASS condition $\left(t_{7}=2.5, P<0.05\right)$. No significant difference was observed in MEP amplitude in the extensor muscle $\left(t_{7}=-2.2, P>0.05\right)$. In addition, the silent period duration in the flexor muscle was longer in RES $(180 \pm 39.3 \mathrm{~ms})$ than in ASS $\left(138 \pm 32.8 \mathrm{~ms} ; t_{7}=-2.59, P<0.05\right)$. All these results are congruent with those previously reported in the literature (Mathis et al., 1998; Bonnard et al., 2003; Sohn et al., 2003).

\section{CNV during the preparatory period}

As can be appreciated from Fig. 3A, throughout the preparatory period, the averaged topographical maps appeared quite similar for the two conditions. The amplitude of the potential increased slowly on the central electrodes during the preparatory period, especially so during the last $2 \mathrm{~s}$ preceding TMS. The amplitude was maximum for the central electrodes from $\mathrm{FC} 3$ to $\mathrm{FC} 2$, and from $\mathrm{C} 3$ to $\mathrm{C} 2$. However, for some electrodes, $\mathrm{CNV}$ amplitude differed between the conditions.

Indeed, an ANOVA yielded a global effect of the condition on $\mathrm{CNV}$ amplitude $\left(F_{1,7}=7.8, P<0.05\right)$. Pairewise comparisons showed that CNV amplitude was significantly lower in the RES than ASS condition for electrodes $\mathrm{FC} 1, \mathrm{C} 1$ and $\mathrm{Cz}\left(q_{7}=3.16,3.2\right.$ and 4.9, respectively, all $P<0.05$ ).

\section{$\alpha$-Oscillations during the preparatory period}

Figure 4A shows the mean spectrogram computed on electrode $\mathrm{C} 3$ observed in one subject during the preparatory period. Figure 4B shows the group-mean $\alpha$-ERD in each condition. Following the instruction, a clear $\alpha$-ERD appeared, after which the oscillation power stabilized during the rest of the preparatory period. As shown in Fig. $4 \mathrm{C}$, maximal $\alpha$-ERDs appeared on electrodes C5, C3, C1, CP5, $\mathrm{CP} 3$ and $\mathrm{CP} 1$.

The ANOVA conducted on the mean $\alpha$-power observed on these electrodes during the last second prior to the IC and to the TMS yielded a significant effect of time-period $\left(F_{1,7}=6.53, P<0.05\right)$. Pairwise comparisons showed that $\alpha$-power was greater during the preinstruction period than during the pre-TMS period for electrodes C5, C3, CP5, CP3 and CP1 (all $P<0.05$ ). However, $\alpha$-power was not different according to condition $\left(F_{1,7}=4.3, P>0.05\right)$, even with planned comparisons restricted to pre-TMS data $\left(F_{1,7}=1.4\right.$, $P>0.05)$.

\section{Brain response to TMS}

Figure $3 \mathrm{~B}$ shows the ERPs observed in the RES and ASS conditions for four central electrodes. They all show a clear TMS-evoked N100. The ANOVA conducted over the N100 amplitude yielded a global effect of condition $\left(F_{1,7}=33.2, P<0.05\right)$ and an effect of electrode $\left(F_{6,42}=4.89, P<0.05\right)$. Pairwise comparisons indicated that $\mathrm{N} 100$ amplitude was significantly greater in the RES than in ASS condition for electrodes $\mathrm{FCz}, \mathrm{Cz}, \mathrm{C} 1, \mathrm{CP} 1$ and $\mathrm{CP} 3\left(q_{7}=3.98,7.02,5.8,3.39\right.$ and 7.14, respectively, all $P<0.05$ ).

Moreover, for $\mathrm{Cz}$, in order to investigate further the relationship between the anticipatory change in excitability and the inhibitory processes, we calculated the correlation between $\mathrm{CNV}$ amplitude and N100 amplitude across all trials for each subject. In six of eight subjects, we found a significant negative correlation between CNV amplitude and N100 amplitude (Pearson product moment correlation coefficient between -0.18 and -0.43 , number of observations between 102 and 188, all $P<0.05$, see Fig. 5).

\section{Discussion}

The present experiment was designed to study how prior intention can tune the excitability of the primary sensorimotor cortex. We used the ability of human subjects to prepare themselves cognitively to resist a TMS-evoked movement by anticipatory selective modulation of corticospinal excitability (Bonnard et al., 2003; Sohn et al., 2003; Camus et al., 2004). We first verified that the muscle response to TMS in the flexor muscle corresponded to previous results in the literature, which was indeed the case: the MEP was decreased and the SP lasted longer in the RES condition than in the ASS condition. Then, based on combined TMS-EEG, we investigated how the sensorimotor cortical networks are involved both during the preparatory period and in the subsequent response to TMS. We found three main results. (i) During the preparatory period, the $\mathrm{CNV}$ amplitude was smaller over central electrodes ipsilateral to TMS (FC1, C1, Cz) when subjects prepared to resist compared with assist the evoked movement. In contrast, $\alpha$-oscillation power was similar in both conditions. (ii) Following TMS, the amplitude of the TMS-evoked N100 component was higher in the RES than in the ASS condition over central electrodes ipsilateral to TMS (FCz, C1, Cz, CP1 and CP3). (iii) Over Cz, in six out of eight subjects, a negative correlation between CNV amplitude and N100 amplitude was found. These results provide clear insight into the mechanisms by which prior intention influences the excitability of the primary motor cortex, as will be discussed now.

\section{Preparation to TMS: effect of prior intention on CNV and $\alpha$-oscillations}

CNV is known to reflect an anticipatory increase in cortical excitability (Bastiaansen et al., 1999; Brunia \& van Boxtel, 2001; Babiloni et al., 2003). The CNV late wave seems to be a mixture of activity related to movement preparation and of activity related to the expectation of an upcoming imperative stimulus (Brunia \& van Boxtel, 2001). Here, the stimuli were the same in both conditions, and thus the observed difference in $\mathrm{CNV}$ can only be attributed to the prepared motor response to TMS. Topographical CNV maps observed here showed that $\mathrm{CNV}$ is maximal for central electrodes from $\mathrm{FC} 3$ to $\mathrm{FC} 2$ and from $\mathrm{C} 3$ to $\mathrm{C} 2$, as also observed during the preparation of stimulus-triggered voluntary movement in a $\mathrm{CNV}$ paradigm (Cui et al., 2000; Filipović et al., 2001; Jankelowitz \& Colebatch, 2002; Babiloni et al., 2004, 2005). Throughout the preparatory period, the overall similarity of these topographical maps of $\mathrm{CNV}$ for the present two experimental conditions strongly suggests that similar cortical networks are involved. However, the CNV amplitude quantitatively differed in the two conditions, the CNV being smaller in the RES condition than in the ASS condition for electrodes FC1, C1 and Cz. This decreased CNV amplitude in the RES condition can be interpreted in terms of a reduced excitability compared with the ASS condition (Babiloni et al., 2003, 2005). Indeed, from a physiological point of view, measuring a negative slow potential at the scalp supposedly reflects the depolarization at the dendrictic tree of the cortical neurons (Birbaumer, 1997; Bastiaansen et al., 1999; Brunia \& van Boxtel, 2001). As a result, 

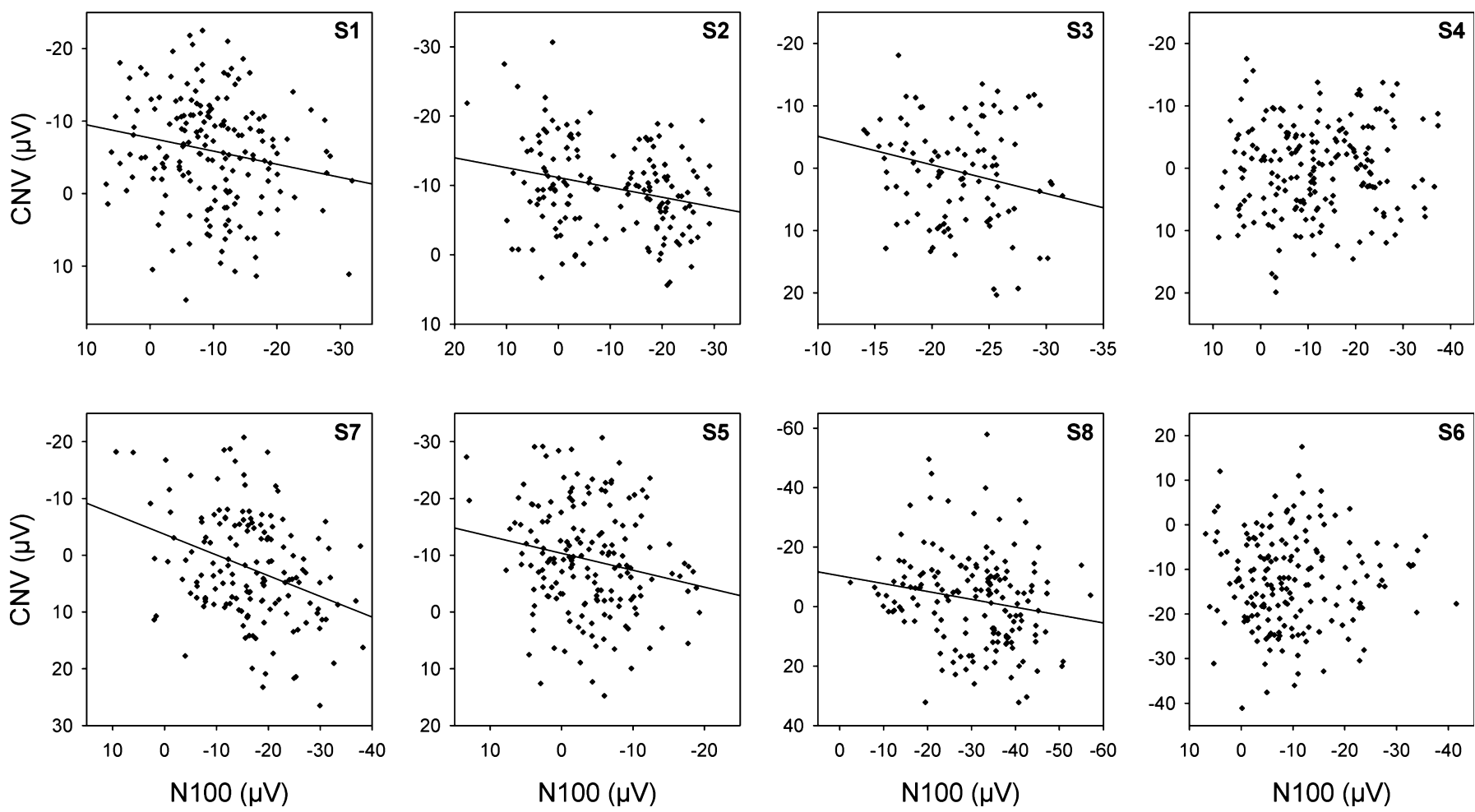

FIG. 5. CNV amplitude plotted against the N100 amplitude observed over Cz for all individual trials regardless of instruction for all eight subjects. Each point represents one trial. For the six subjects showing a significant correlation between these two variables (Pearson test), the regression line is represented as a solid line.

the membrane potential of these cells gets closer to their firing threshold, which increases their readiness to fire (Bastiaansen et al., 1999). For our present result, this strongly suggests that the sensorimotor cortex had a lower excitability in the RES than in the ASS condition prior to TMS.

$\alpha$-Oscillations are thought to be generated by thalamo-cortical loops (Lopes da Silva \& Pfurtscheller, 1999). Power modulation of $\alpha$-oscillations is considered to be linked to cortical activation/deactivation (Filipović et al., 2001; Babiloni et al., 2003; Neuper et al., 2006). A decrease in $\alpha$-band oscillatory activity ( $\alpha$-ERD) is associated with an activated state of the underlying area (Lopes da Silva \& Pfurtscheller, 1999; Filipović et al., 2001). In line with this hypothesis, $\alpha$-ERD over the sensorimotor cortex has been reported before both self-paced and externally paced movements (Stančák et al., 2000; Filipović et al., 2001). Here, we observed an $\alpha$-ERD following the instruction, and a stabilization of the $\alpha$-power during the subsequent preparatory period, which shows that the preparation of the motor reaction to TMS is associated with sensorimotor cortex activation. At the end of the preparatory period, however, no difference in $\alpha$-power was observed between the two conditions, suggesting that the task to resist vs. assist a TMS-evoked movement is not realized by a modulation of cortical activation. One might have expected the opposite because spontaneous fluctuations of cortical oscillations have been found to co-vary with corticospinal excitability (Zarkowski et al., 2006; Sauseng et al., 2009). Indeed, these studies showed that if TMS around the motor threshold is applied over M1 during spontaneous $\alpha$-oscillations, MEP amplitude is related to $\alpha$-power: decreased $\alpha$-oscillation power is associated with increased MEP amplitude. However, in our study, subjects were engaged in a motor task in both conditions, and MEP amplitude modulations were not associated with modulation of $\alpha$-oscillation power. This is in line with a study from
Lepage et al. (2008), who investigated the correlation between $\alpha$-power modulations and MEP amplitude during three tasks (execution, visualization or observation of a movement). Compared with a rest condition, $\alpha$-oscillation power was decreased and MEP amplitude was increased for all three conditions. However, no correlation was established between $\alpha$-oscillation power and MEP amplitude across the experimental conditions because MEP amplitude was greater during the execution than during the visualization or the observation conditions while $\alpha$-oscillation power was similar in all three conditions. Furthermore, in reaction time paradigms, Leocani et al. (2001) also pointed out that $\alpha$-ERD is compatible with either an increase or a decrease in CS excitability. While $\alpha$-ERD is bilateral during movement execution, CS excitability is decreased on the side ipsilateral to movement and increased on the controlateral side. Together, these results are consistent with the present results and argue against a relationship between CS excitability and $\alpha$-ERD during motor tasks. Thus, contrary to what happens when no task is required and $\alpha$ oscillations fluctuate spontaneously, when subjects are engaged in a motor task, modulations of CS excitability are not associated with modulations of $\alpha$-oscillations.

For the preparatory period, while CNV differed between conditions, $\alpha$-oscillation power did not. The same pattern of results indicating an uncoupling of CNV and $\alpha$-ERD has already been reported in a Go-NoGo task (Filipović et al., 2001). The present results show that the anticipatory processing in the sensorimotor cortex allowing one to resist vs. assist a TMS-evoked movement does not induce modulation of cortical activation (as shown by the absence of $\alpha$-power difference) but rather induces a change in cortical excitability (as shown by the modulation of CNV). So far, no hypothesis about the involvement of excitatory/inhibitory networks giving rise to this lower excitability can be drawn. 


\section{Reaction to TMS: effect of prior intention on the evoked brain response}

The different preparatory motor sets prior to TMS, which presumably decrease the sensorimotor cortex excitability more in the RES than in the ASS condition (as shown by the smaller CNV observed in the former condition), directly influenced the cortical TMS-evoked response. Following TMS, the amplitude of the N100 component was higher in the RES than in the ASS condition on some central electrodes ( $\mathrm{FCz}, \mathrm{Cz}, \mathrm{C} 1, \mathrm{CP} 1$ and $\mathrm{CP} 3)$. Although small, this difference was significant, as with those reported by Nikulin et al. (2003) or Bender et al. (2005). Moreover, while both abovementioned studies compared a movement condition with a no-movement condition, in the present experiment, the subjects always maintained a given hand posture against gravity with similar ongoing EMG activity, and they had to produce a motor response to TMS albeit a different one in the two conditions. The difference in the N100-evoked response could thus only be due to the different prepared and executed reaction.

The inhibitory nature of the N100 component following TMS has been well documented (Nikulin et al., 2003; Bender et al., 2005; Kičić et al., 2008). Indeed, these studies showed that the amplitude of the N100 component of the TMS-evoked response was smaller when applied immediately before movement onset (during the increased premovement cortical excitability) in comparison with a no-movement condition (Nikulin et al., 2003; Bender et al., 2005). Thus, an attenuation of the N100 component has been hypothesized to indicate an increased level of cortical excitability (Nikulin et al., 2003; Bender et al., 2005; Kičić et al., 2008). Indirect evidence for the inhibitory nature of the N100 also comes from the paired-pulse TMS paradigm using a suprathreshold conditioning stimulus, showing a peak in the attenuation of the conditioned MEPs at 100-150 ms following the conditioning stimulus presentation (Valls-Solé et al., 1992; Nakamura et al., 1997). Moreover, using combined TMS-EEG, recent studies have showed that paired-pulse TMS using suprathreshold conditioning and test stimuli with inter-stimuli intervals of $100 \mathrm{~ms}$ resulted in a significant suppression of mean cortical-evoked activity in the motor cortex (Daskalakis et al., 2008; Fitzgerald et al., 2008). This strongly suggests that the N100-evoked response reflects inhibitory processes. Several studies (Nikulin et al., 2003; Kičić et al., 2008) have proposed that the N100 component evoked by TMS corresponds to the longlasting inhibitory postsynaptic potentials (IPSPs $>100 \mathrm{~ms}$ ) observed in intracellular recordings following brain surface electrical stimulation (Krnjević et al., 1966; Rosenthal et al., 1967).

The present results offer a clear validation of the hypothesis that an increased N100 amplitude reflects a decreased cortical excitability due to increased inhibitory processes. Indeed, because we compared two conditions in which the subject had to prepare and execute two different motor responses to TMS, we now have several converging arguments. First, in the RES condition, the N100 component amplitude was increased while the CNV before TMS occurrence was decreased relative to the ASS condition; moreover, following TMS the SP lasted longer in the resist condition. Whereas the reduced $\mathrm{CNV}$ reveals a decreased cortical excitability, the longer SP strongly suggests an increase in inhibitory processes in the motor cortex in the RES condition. Indeed, the last part of the SP results from the excitation of inhibitory interneurons of the motor cortex (Abbruzzese \& Trompetto, 2002; Terao \& Ugawa, 2002). Therefore, the duration of the SP reflects the degree of cortical inhibition. Such modulations of the SP duration related to the instruction set had already been observed in protocols similar to ours (Mathis et al., 1998; Hoshiyama \& Kakigi, 1999) but they have previously not been related to differences in cortical states.
Secondly, and more importantly, the negative correlation between CNV amplitude and that of the TMS-evoked N100 component allows us to demonstrate the relationship between the anticipatory change in excitability and the inhibitory processes. This strongly supports the hypothesis that in the resist condition the decreased cortical excitability is due to increased inhibitory processes. From this perspective, the differential modulation of inhibitory processes would be one mechanism underlying the distinct change in cortical excitability in both conditions. However, this does not exclude the existence of other mechanisms (subcortical among others). Thus, the present results together show that when subjects prepare to resist TMS, the anticipatory processes cause a decrease in the excitability by increasing inhibitory processes at the cortical level. It is likely that the change in cortical excitability in the resist condition compared with the assist condition is not global (affecting a large cortical area and all innervated muscles) but rather very local. Indeed, muscle recordings showed that this modulation in cortical excitability was accompanied by a selective decrease in CS excitability affecting only the wrist flexor muscle, and not the extensor muscle (Bonnard et al., 2003; Sohn et al., 2003; Camus et al., 2004). This selectivity in the muscle response to TMS argues in favour of a very local suppression.

\section{Conclusions}

Using combined EEG-TMS during cognitive preparation of a reaction to TMS, we directly showed that prior intention can tune the excitability of the primary motor cortex before any peripheral influence. Thereby, we revealed some cortical mechanisms underlying the interaction between cognitive and motor function by showing the relationship between the anticipatory change in cortical excitability (as revealed by the CNV) and the cortical inhibitory processes (as revealed by the TMSevoked N100 component). Our three findings all point to the same conclusion: when subjects prepare to resist a TMS-evoked movement, the anticipatory processes cause a decrease in the cortical excitability by increasing inhibitory processes. Indeed, comparing the resist to the assist conditions, the amplitude of the CNV preceding TMS decreased while the amplitude of the TMS-evoked N100 component increased. Moreover, both amplitude correlated negatively. The higher amplitude of the N100 component in the resist condition favours an active inhibitory process. Interestingly, the decrease in cortical excitability in the resist condition appeared to correspond to a very local inhibition rather than a global decrease in excitation.

Preparing a voluntary reaction to TMS was found to engage cortical integrative processes in sensorimotor networks which depend on the prepared response. Interestingly, these results resemble those observed by Evarts \& Tanji (1974) in the case of preparatory motor set anticipating a mechanical perturbation (instead of a central perturbation in the present experiment). These authors showed that when an animal anticipates a peripheral movement perturbation, motor cortex activity is modulated according to the prepared reaction. However, future research is needed to determine whether the cerebral mechanisms involved in preparing a reaction to a central vs. peripheral perturbation are similar.

\section{Acknowledgments}

This research was supported by the French Centre National de la Recherche Scientifique and Aix-Marseille University. We warmly thank Raoul Huys and Gita Prabhu for many helpful comments on an earlier version of this manuscript. Finally, we would like to thank both reviewers for very helpful comments and suggestions. 


\section{Abbreviations}

ASS, assist; CNV, contingent negative variation; $\mathrm{CS}$, corticospinal; ECR extensor carpi radialis; EEG, electroencephalographic; EMG, electromyographic; ERD, event-related desynchronization; ERP, event-related potential; FCR, flexor carpi radialis; IC, instruction cue; IPSP, inhibitory post-synaptic potential; M1, primary motor cortex; MEP, motor evoked potential; MRI, magnetic resonance imaging; RES, resist; SP, silent period; TMS, transcranial magnetic stimulation.

\section{References}

Abbruzzese, G. \& Trompetto, C. (2002) Clinical and research methods for evaluating cortical excitability. J. Clin. Neurophysiol., 19, 307-321.

Babiloni, C., Brancucci, A., Babiloni, F., Capotosto, P., Carducci, F., Cincotti, F., Arendt-Nielsen, L., Chen, A.C. \& Rossini, P.M. (2003) Anticipatory cortical responses during the expectancy of a predictable painful stimulation. A highresolution electroencephalography study. Eur. J. Neurosci., 18, 1692-1700.

Babiloni, C., Brancucci, A., Arendt-Nielsen, L., Del Percio, C., Babiloni, F., Pascual-Marqui, R.D., Sabbatini, G., Rossini, P.M. \& Chen, A.C. (2004) Cortical sensorimotor interactions during the expectancy of a go/noGo task: effect of painful stimuli. Behav. Neurosci., 118, 925-935.

Babiloni, C., Brancucci, A., Capotosto, P., Romani, G.L., Arendt-Nielsen, L., Chen, A.C. \& Rossini, P.M. (2005) Slow cortical potential shifts preceding sensorimotor interactions. Brain Res. Bull., 65, 309-316.

Bastiaansen, M., Brunia, C. \& Böcker, K. (1999) ERD as an index of anticipatory behavior. In Pfurtscheller, G. \& Lopez da Silva, F.H. (Eds), Event-Related Desynchronization, Handbook of Electroencephalography and Clinical Neurophysiology, revised series, vol 6. Elsevier Science BV, Amsterdam, pp. 203-217.

Bender, S., Basseler, K., Sebastian, I., Resch, F., Kammer, T., Oelkers-Ax, R. \& Weisbrod, M. (2005) Electroencephalographic response to transcranial magnetic stimulation in children: evidence for giant inhibitory potentials. Ann. Neurol., 58, 58-67.

Birbaumer, N. (1997) Slow cortical potentials: their origin, meaning and clinical use. In van Boxtel, G. \& Böcker, K. (Eds), Brain and Behavior: Past, Present and Future. Tilburg University Press, Tilburg, pp. 25-41.

Bonnard, M., Camus, M., de Graaf, J. \& Pailhous, J. (2003) Direct evidence for a binding between cognitive and motor functions in humans: a TMS study. J. Cogn. Neurosci., 15, 1207-1216.

Bonnard, M., de Graaf, J. \& Pailhous, J. (2004) Interactions between cognitive and sensorimotor functions in the motor cortex: evidence from the preparatory motor sets anticipating a perturbation. Rev. Neurosci., 15, 371-382.

Brunia, C.H. \& van Boxtel, G.J. (2001) Wait and see. Int. J. Psychophysiol., 43, 59-75.

Camus, M., Pailhous, J. \& Bonnard, M. (2004) Cognitive tuning of the corticospinal excitability during human gait: a TMS study. Eur. J. Neurosci., 20, 1101-1107.

Cui, R.Q., Egkher, A., Huter, D., Lang, W., Lindinger, G. \& Deecke, L. (2000) High resolution spatiotemporal analysis of the contingent negative variation in simple or complex motor tasks and a non-motor task. Clin. Neurophysiol., 111, 1847-1859.

Daskalakis, Z.J., Farzan, F., Barr, M.S., Maller, J.J., Chen, R. \& Fitzgerald, P.B. (2008) Long-interval cortical inhibition from the dorsolateral prefrontal cortex: a TMS-EEG study. Neuropsychopharmacology, 33, 2860-2869.

Day, B.L., Riescher, H., Struppler, A., Rothwell, J.C. \& Marsden, C.D. (1991) Changes in the response to magnetic and electrical stimulation of the motor cortex following muscle stretch in man. J. Physiol. Lond., 433, 41-57.

Evarts, E.V. \& Tanji, J. (1974) Gating of motor cortex reflexes by prior instruction. Brain Res., 71, 479-494.

Evarts, E.V., Shinoda, Y. \& Wise, S.P. (1984) Neurophysiological Approaches to Higher Brain Functions. John Wiley \& Sons, New-York.

Filipović, S.R., Jahanshahi, M. \& Rothwell, J.C. (2001) Uncoupling of contingent negative variation and alpha band event-related desynchronization in a go/no-go task. Clin. Neurophysiol., 112, 1307-1315.

Fitzgerald, P.B., Daskalakis, Z.J., Hoy, K., Farzan, F., Upton, D.J., Cooper, N.R. \& Maller, J.J. (2008) Cortical inhibition in motor and non motor regions: a combined TMS-EEG study. Clin. EEG Neurosci., 39, 112-117.

Georgopoulos, A.P. (2000) Neural aspects of cognitive motor control. Curr. Opin. Neurobiol., 10, 238-241.

Hoshiyama, M. \& Kakigi, R. (1999) Shortening of the cortical silent period following transcranial magnetic brain stimulation during an experimental paradigm for generating contingent negative variation (CNV). Clin. Neurophysiol., 110, 1394-1398.
Ilmoniemi, R.J., Virtanen, J., Ruohonen, J., Karhu, J., Aronen, H.J., Näätänen, R. \& Katila, T. (1997) Neuronal responses to magnetic stimulation reveal cortical reactivity and connectivity. Neuroreport, 8, 3537-3540.

Jackson, A., Spinks, R.L., Freeman, T.C., Wolpert, D.M. \& Lemon, R.N. (2002) Rhythm generation in monkey motor cortex explored using pyramidal tract stimulation. J. Neurophysiol., 541, 685-699.

Jankelowitz, S.K. \& Colebatch, J.G. (2002) Movement-related potentials associated with self-paced, cued and imagined arm movements. Exp. Brain Res., 147, 98-107.

Kičić, D., Lioumis, P., Ilmoniemi, R.J. \& Nikulin, V.V. (2008) Bilateral changes in excitability of sensorimotor cortices during unilateral movement: Combined electroencephalographic and transcranial magnetic stimulation study. Neuroscience, 152, 1119-1129.

Kimura, T., Haggard, P. \& Gomi, H. (2006) Transcranial magnetic stimulation over sensorimotor cortex disrupts anticipatory reflex gain modulation for skilled action. J. Neurosci., 26, 9272-9281.

Krnjević, K., Randić, M. \& Straughan, D.W. (1966) An inhibitory process in the cerebral cortex. J. Physiol., 184, 16-48.

Leocani, L., Toro, C., Zhuang, P., Gerloff, C. \& Hallett, M. (2001) Event-related desynchronization in reaction time paradigms: a comparison with event-related potentials and corticospinal excitability. Clin. Neurophysiol., 112, 923-930.

Lepage, J.F., Saint-Amour, D. \& Théoret, H. (2008) EEG and neuronavigated single-pulse TMS in the study of the observation/execution matching system: are both techniques measuring the same process? J. Neurosci. Methods, 175, 17-24.

Lopes da Silva, F.H. \& Pfurtscheller, G. (1999) Basic concept on EEG synchronization and desynchronization. In Pfurtscheller, G. \& Lopez da Silva, F.H. (Eds), Event-Related Desynchronization, Handbook of Electroencephalography and Clinical Neurophysiology, revised series, vol 6. Elsevier Science BV, Amsterdam, pp. 3-11.

Massimini, M., Ferrarelli, F., Huber, R., Esser, S.K., Singh, H. \& Tononi, G. (2005) Breakdown of cortical effective connectivity during sleep. Science, 309, 2228-2232.

Mathis, J., de Quervain, D. \& Hess, C.W. (1998) Dependence of the transcranially induced silent period on the 'instruction set' and the individual reaction time. Electroencephalogr. Clin. Neurophysiol., 109, 426-435.

Meyer-Lindenberg, A., Ziemann, U., Hajak, G., Cohen, L. \& Berman, K.F. (2002) Transitions between dynamical states of differing stability in the human brain. Proc. Natl. Acad. Sci. USA, 99, 10948-10953.

Nakamura, H., Kitagawa, H., Kawaguchi, Y. \& Tsuji, H. (1997) Intracortical facilitation and inhibition after transcranial magnetic stimulation in conscious humans. J. Physiol., 498, 817-823.

Neuper, C., Wörtz, M. \& Pfurtscheller, G. (2006) ERD/ERS patterns reflecting sensorimotor activation and deactivation. Prog. Brain Res., 159, 211-222.

Nikulin, V.V., Kičić, D., Kähkönen, S. \& Ilmoniemi, R.J. (2003) Modulation of electroencephalographic responses to transcranial magnetic stimulation: evidence for changes in cortical excitability related to movement. Eur. J. Neurosci., 18, 1206-1212.

Palmer, E. \& Ashby, P. (1992) Evidence that a long latency stretch reflex in humans is transcortical. J. Physiol. Lond., 449, 429-440.

Paus, T., Sipilä, P.K. \& Strafella, A.P. (2001) Synchronization of neuronal activity in the human primary motor cortex by transcranial magnetic stimulation: an EEG study. J. Neurophysiol., 86, 1983-1990.

Pfurtscheller, G. \& Lopes da Silva, F.H. (1999) Event-related EEG/MEG synchronization and desynchronization: basic principles. Clin. Neurophysiol., 110, 1842-1857.

Requin, J., Brener, J. \& Ring, C. (1991) Preparation for action. In Jennings, J.R. \& Coles, M.G. (Eds), Handbook of Cognitive Psychophysiology: Central and Autonomic Nervous System Approaches. John Wiley \& Sons, New-York, pp. $357-448$.

Riehle, A. (2004) Preparation for action: one of the key functions of motor cortex. In Riehle, A. \& Vaadia, E. (Eds), Motor Cortex in Voluntary Movements. CRC press, Boca Raton, FL, pp. 213-240.

Rosenthal, J., Waller, H.J. \& Amassian, V.E. (1967) An analysis of the activation of motor cortical neurons by surface stimulation. J. Neurophysiol., 30, $844-858$.

Rothwell, J.C., Traub, M.M. \& Marsden, C.D. (1982) Automatic and 'voluntary' responses compensating for disturbances of human thumb movement. Brain Res., 248, 33-41.

Rumeau, C., Tzourio, N., Murayama, N., Peretti-Viton, P., Levrier, O., Joliot, M., Mazoyer, B. \& Salamon, G. (1994) Location of hand function in the sensorimotor cortex: MR and functional correlation. Am. J. Neuroradiol., 15, $567-572$.

Sastre-Janer, F.A., Regis, J., Belin, P., Mangin, J.-F., Dormont, D., Masure, M.-C., Remy, P., Froulin, V. \& Samson, Y. (1998) Three-dimensional 
reconstruction of the human central sulcus reveals a morphological correlate of the hand area. Cereb. Cortex, 8, 641-647.

Sauseng, P., Klimesch, W., Gerloff, C. \& Hummel, F.C. (2009) Spontaneous locally restricted EEG alpha activity determines cortical excitability in the motor cortex. Neuropsychologia, 47, 284-288.

Schieppati, M., Trompetto, C. \& Abbruzzese, G. (1996) Selective facilitation of responses to cortical stimulation of proximal and distal arm muscles by precision tasks in man. J. Physiol. (London), 491, 551-562.

Sohn, Y.H., Dang, N. \& Hallett, M. (2003) Suppression of corticospinal excitability during negative motor imagery. J. Neurophysiol., 90, 2303-2309.

Stančák, A.J., Feige, B., Lücking, C.H. \& Kristeva-Feige, R. (2000) Oscillatory cortical activity and movement-related potentials in proximal and distal movements. Clin. Neurophysiol., 111, 636-650.
Tanji, J. \& Evarts, E.V. (1976) Anticipatory activity in motor cortex neurons in relation to direction of an intented movement. J. Neurophysiol., 39, 1062-1068.

Terao, Y. \& Ugawa, Y. (2002) Basic mechanisms of TMS. J. Clin. Neurophysiol., 19, 322-343.

Valls-Solé, J., Pascual-Leone, A., Wassermann, E.M. \& Hallett, M. (1992) Human motor evoked responses to paired transcranial magnetic stimuli. Electroencephalogr. Clin. Neurophysiol., 85, 355-364.

Yousry, T.A., Schmid, U.D., Alkadhi, H., Schmidt, D., Peraud, A., Buettner, A. \& Winkler, P. (1997) Localization of the motor hand area to a knob on the precentral gyrus. Brain, 120, 141-157.

Zarkowski, P., Shin, C.J., Dang, T., Russo, J. \& Avery, D. (2006) EEG and the variance of motor evoked potential amplitude. Clin. EEG Neurosci., 37, $247-251$. 